\title{
Automatic Selection and Combination of Descriptors for Effective 3D Similarity Search
}

\author{
Benjamin Bustos Daniel Keim Dietmar Saupe Tobias Schreck Dejan Vranić \\ Department of Computer and Information Science, University of Konstanz \\ \{bustos,keim,saupe,schreck,vranic\}@informatik.uni-konstanz.de
}

\begin{abstract}
We focus on improving the effectiveness of similarity search in $3 D$ object repositories from a system-oriented perspective. Motivated by an effectiveness evaluation of several individual $3 D$ retrieval methods, we research a selection heuristic, called purity, for choosing retrieval methods based on query-dependent characteristics. We show that the purity selection method significantly improves the search effectiveness compared to the best single methods. We then show that retrieval effectiveness can be further boosted by considering combinations of multiple retrieval methods to perform the search. We propose to use a dynamically weighted combination of feature vectors based on the purity concept, and we experimentally show that the search effectiveness of our combined methods by far exceeds the effectiveness of our best implemented single method.
\end{abstract}

Keywords: 3D objects, information retrieval, query by content, effectiveness, feature selection.

\section{Introduction}

The development of effective content-based multimedia search systems is an important research issue due to the growing amount of digital audio-visual information. In the case of images and video, the growth of digital data has been observed since the introduction of 2D capture devices. A similar development is expected for 3D data, as acquisition and dissemination technology is constantly improving. In digital libraries, it is possible to search using annotation information, which describes the content of an object in textual form, or using the multimedia data itself, the so-called content-based search. The latter is the more promising approach, because in general textual descriptions are manually created, which is prohibitively expensive, and they are subject to the opinion of the person who creates them. In contrast, content-based search algorithms allow an implementation of fully automatic retrieval systems.
There are many practical applications of similarity search in 3D libraries. In medicine, the detection of similar organ deformations can be used for diagnostic purposes. The search of similar standard parts can help in reducing costs in the manufacturing industry. There are also applications in the entertainment industry, e.g., film production and video games. Figure 1 illustrates the concept of content-based 3D similarity search. (q)

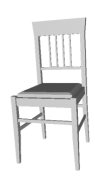

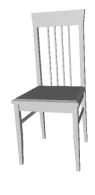

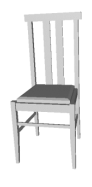

(a)

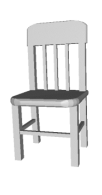

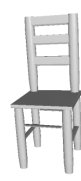
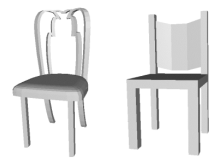

Figure 1. Example of a similarity query in a 3D object database, showing a query object (q) and a set of possibly relevant objects (a).

In this paper, we experimentally compare a range of 3D feature vectors (FVs), and we propose methods for improving the effectiveness of the 3D similarity search process. Our new method, called purity selection, determines which FV should be used depending on the query object. This leads to a significant improvement in retrieval effectiveness compared with the best single FV. Also, we propose methods for FVs, which leads to further significant improvements of the effectiveness of the search system. Our experimental results show that the relative ordering of FVs by retrieval effectiveness depends on the query point, which means that no single FV outperforms all other FVs on all queries, and that linear combinations of multiple FVs provide a significant improvement of the retrieval effectiveness compared to the best single FV. 


\section{Similarity search of 3D objects}

\subsection{Feature-based approach for $3 D$ retrieval}

Usually, a feature vector approach is adopted for performing similarity search in multimedia databases. The basic idea is to derive a vector of numerical (real) values for each of the objects in the repository, extracting those properties of the 3D objects that best support an applicationdependent notion of similarity. For a given feature extraction technique, it is usually possible to generate FVs of different dimensionality by setting the resolution with which the FV extraction proceeds. FVs describing 3D objects may be derived from object geometry and/or other attributes and should be invariant to changes in the orientation (translation, rotation and reflection) and scale of 3D models. Good FVs should also be robust with respect to small changes in the level-of-detail, geometry and topology of the models.

Given the FVs for the objects in a database and a query point, the retrieval of similar objects is performed by returning the $k$ nearest neighbors $(k-\mathrm{NN})$ of the query point. To this end, a metric in the vector space $\mathbb{R}^{d}$ (for dimensionality $d$ depending on parameterization of the FV extraction method at hand), is used, e.g., the unweighted Minkowski $\left(l_{p}\right)$ distance, given by $l_{p}(\vec{x}, \vec{y})=\left(\sum_{1 \leq i \leq d}\left|x_{i}-y_{i}\right|^{p}\right)^{1 / p}, p \geq 1$. More sophisticated metrics for vector spaces, e.g., quadratic forms [2], exist, but their applicability depends on the FV definition and computational efficiency considerations.

\subsection{Related work and studied feature vectors}

The last few years have seen a strongly increasing interest in content-based retrieval of 3D models, and its popularity may be expected to eventually become as large as the popularity of similarity search in image databases. Algorithms have been proposed to extract FVs based on many different object characteristics. Statistical FVs include geometric moments [22, 17, 15, 20], and histograms of measures like the distribution of distances between points on an object's surface [16]. Some of the extention-based methods treat 3D objects as functions defined on spheres, and describe the objects in terms of samples taken from these functions [26, 21, 14]. Many algorithms derive object descriptions from certain space partitioning schemes $[1,11,23,5,19,13]$. Furthermore, the curvature of an object's surface may be considered like in [27] or [10]. FVs may also be obtained from 2D renderings of the objects as in $[7,4]$. There also exist non-FV approaches to 3D retrieval, which rely on topological [8] or skeletal descriptions of the models [18]. Considering the specific problem of securing rotation invariance of the description, there exists an ongoing discussion whether this should be achieved by the appli- cation of a rotation normalization step prior to feature calculation [21], or by the definition of FVs that are implicitly rotational invariant [12]. In our work, we consider FVs that rely on rotation normalization using a variant of the Principal Component Analysis (PCA) [26], as we believe it is stable in many cases, and contributes valuable information to the object description.

While we have implemented many different FVs from our own as well as other researchers work in our 3D similarity search system, for clarity reasons we focus the remainder of this paper to a set of six algorithms which belong to the FVs providing the best retrieval quality in our experiments. Specifically, we consider two FVs based on the Fourier transform of rendered silhouettes and Z-buffers of the 3D models, resulting in the silhouette and depth buffer FVs respectively, as presented in [7]. Also, we consider the spherical harmonics transform of samples taken from model extention, as well as samples taken from a combination of model extention and surface orientation properties, resulting in the ray-based and the complex FVs respectively, as introduced in [25]. Furthermore, we include in this study a FV based on the discretization of model surface in a voxel grid (the voxel FV, as introduced in [24]), and the implicitly rotation invariant algorithm introduced in [5] which is based on the spherical harmonics transform of concentric functions defined on the voxelization of models, and that we would like to call the harmonics $3 D \mathrm{FV}$ in this paper.

\section{Measuring retrieval effectiveness}

\subsection{Description of the experiments and the effec- tiveness measures}

The database used for retrieval experiments contains $1,8373 \mathrm{D}$ objects collected from the Internet. From this set, 472 objects were classified by shape into 55 different model classes, yielding the ground truth, and the rest of them were left as "unclassified". Each classified object was used as a query object, and the objects belonging to the same model class, excluding the query, were considered relevant to it.

Table 1 gives a partial description (first 20 classes) of the classified objects of the database. The first column indicates the class identification number. The second column describes the $3 \mathrm{D}$ class models. The last column lists the number of objects per model class.

For comparing the effectiveness of the search algorithms, we use precision vs. recall figures, a standard evaluation technique for retrieval systems [3]. Precision is the fraction of the retrieved objects which are relevant to a given query, and recall is the fraction of the relevant objects which have been retrieved from the database. In addition, we also consider the widely used $R$-precision measure [3]. It is defined as the precision when retrieving as many objects as 


\begin{tabular}{|c|c|c|}
\hline Class id \# & Description & \# of models \\
\hline \hline 1 & ants & 6 \\
2 & rabbits & 4 \\
3 & cows & 7 \\
4 & dogs & 4 \\
5 & sea animals & 13 \\
6 & bees & 5 \\
7 & CPU's & 4 \\
8 & keyboards & 8 \\
9 & cans & 4 \\
10 & bottles & 14 \\
11 & bowls & 4 \\
12 & pots & 4 \\
13 & cups & 8 \\
14 & wine glasses & 9 \\
15 & teapots & 4 \\
16 & biplanes & 5 \\
17 & helicopters & 9 \\
18 & missiles & 16 \\
19 & jet planes & 18 \\
20 & fighter jet planes & 26 \\
\hline
\end{tabular}

Table 1. Partial description of the classified set of our 3D object database

there are relevant answers in the database, w r.t. the query. We average these measures over all queries that belong to one of the predefined query classes. As the metric of choice we employ the $l_{1}$ (Manhattan) distance, as we experimentally found this gives us the best retrieval results compared to other Minkowski distances $\left(l_{1}\right.$ was slightly but consistently better than $l_{2}$ in our experiments).

\subsection{Results using single feature vectors}

In our first experiments, we compared the retrieval performance of six FVs using our ground truth. To first assess the influence of FV resolution, we evaluated a range of FV dimensionality settings. Figure 2 shows the effect of the FV dimensionality on the overall effectiveness of the FVs, measured in terms of R-precision. The figure shows that effectiveness improvement rate diminishes quickly for roughly more than 64 dimensions for most FVs. It is interesting to note that the saturation effect is reached at roughly the same dimensionality level. This is not an expected result, considering that the different FVs describe different characteristics of the 3D objects.

Figure 3 shows the database-average effectiveness performance (precision vs. recall curves and R-precision values) of the six FVs when using their best dimensionality parameterization, respectively. The best performing FV on av-

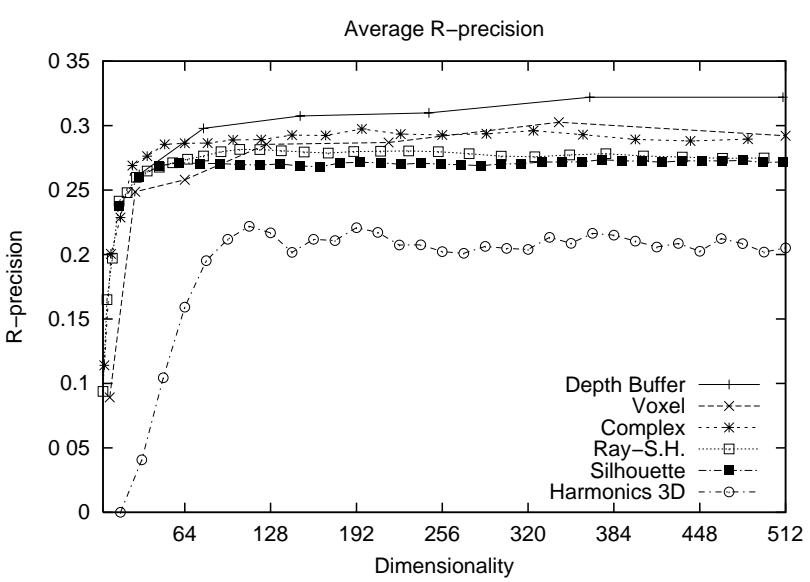

Figure 2. R-precision as a function of the dimensionality of the feature vectors.

erage is the image-based depth buffer FV. Between the $1^{\text {st }}$ and the $3^{r d}$ best $\mathrm{FV}$, the performance differences are small, implying that in practice all these FVs have similar retrieval capabilities.

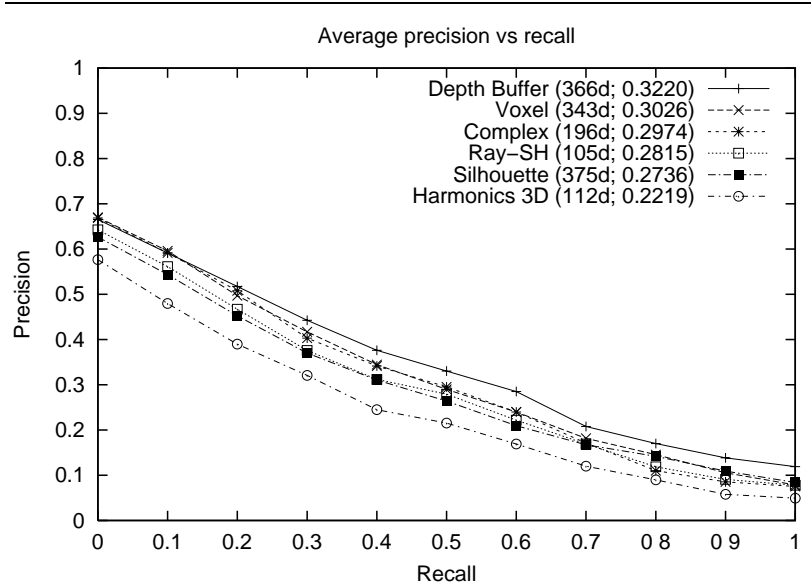

Figure 3. Average precision vs. recall figures for all feature vectors (the legend includes the optimal dimensionality and the Rprecision values).

Now we present the results obtained for two specific model classes. Figure 4 shows the average precision vs. recall figures for the Formula 1 cars model class. In this case, the best effectiveness is obtained with the depth buffer and the harmonics 3D FVs. Note that the best FV for this model class is also the best FV on average. The R-precision value is also given for each FV. 


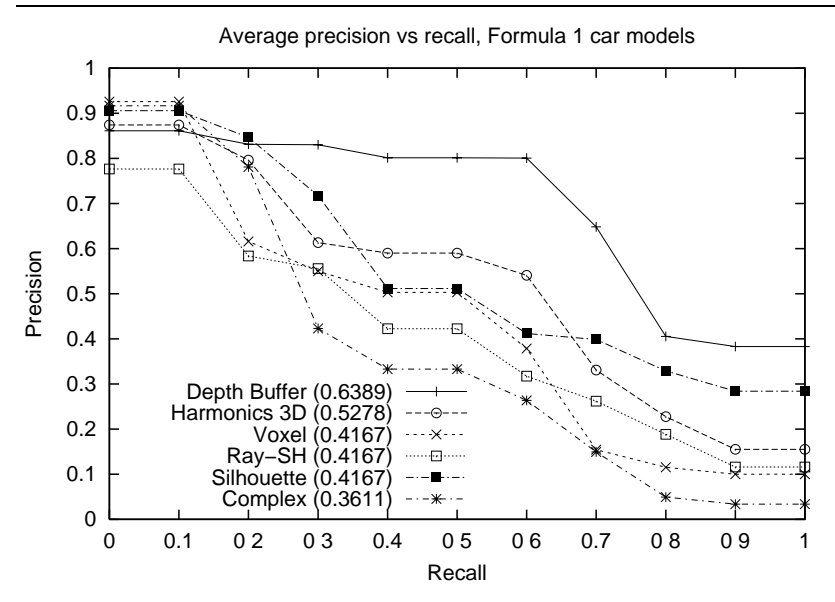

Figure 4. Average precision vs. recall figures and $R$-precision values for the $F-1$ cars model class.

Figure 5 shows the average precision vs. recall figure for the sea animals model class. For this class, the best FVs are the silhouette and the ray-based spherical harmonics FVs. This result shows that for some model classes the best average FV (depth buffer) does not perform well. Moreover, the best three FVs for this class are different from the best three FVs of the F-1 cars model class.

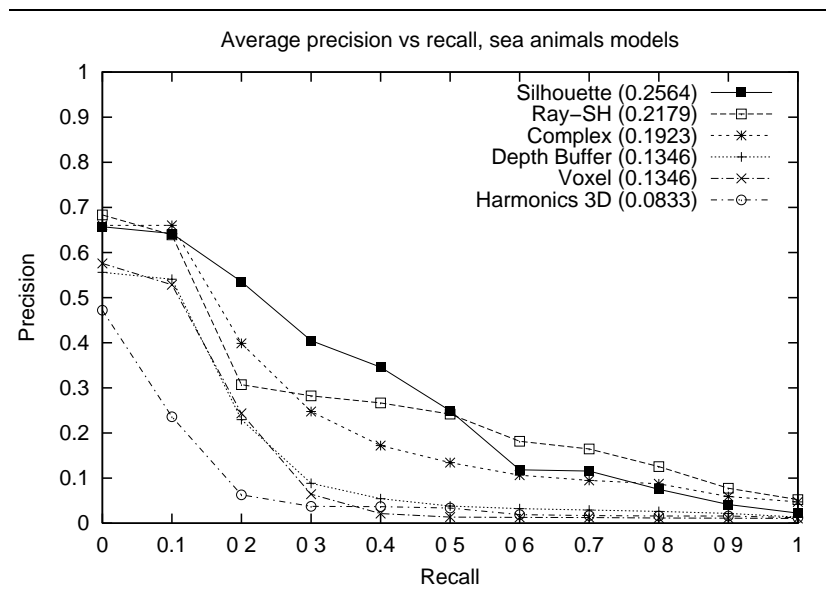

Figure 5. Average precision vs. recall figures and R-precision values for the sea animals model class.

In general, we observed that for many query classes, the respective ranking of FVs by retrieval quality differs from the average ranking. It follows that an appropriate selection of the FV used for the similarity search, depending on the query object, could improve the overall retrieval effective- ness compared with the policy of always choosing a certain default FV.

\section{Purity-based feature selection}

The previous results indicate the need for appropriate feature selection, based on the query to be evaluated. At our disposal is a set of FVs, but how can we automatically estimate the quality of the result when choosing one of them? Note that we want to support similarity queries. In classification, feature selection refers to choosing the features that optimize an objective function (usually, classification accuracy). For similarity search, we first need to find such an objective function estimating the quality of a similarity ranking.

\subsection{Query dependent selection of features}

Let $X$ be the universe of valid 3D objects, $U \subseteq X$ the 3D database, and $Q \subseteq U$ a set of classified objects, that is, $Q=\biguplus_{i=1}^{m} Q_{i}$, where $Q_{i}$ is a model class (i.e., a set of similar objects), and $Q$ is the disjoint union of $m$ model classes.

Definition 1 Given a set of $\ell F V s\left\{f_{1}, \ldots, f_{\ell}\right\}$, a $3 D$ query object $q \in X$, and a constant value $k \in \mathbb{N}^{+}$, we generate $\ell$ object rankings, one for each $F V$, consisting of the distances between $q$ and every object of $Q$ sorted in ascending order. Let $R_{q k}^{j}$ be the first $k$ positions of the ranking using $f_{j}$, and let $S_{i}^{j}=R_{q k}^{j} \cap Q_{i}$. The purity of $f_{j}$ for the query $q$ is defined as:

$$
\operatorname{purity}\left(f_{j}, q, k\right)=\max _{1 \leq i \leq m}\left(\left|S_{i}^{j}\right|\right)
$$

The purity value indicates the maximum number of objects that belong to a same model class in the first $k$ positions of each ranking. The FV that has the maximum purity is selected for performing the search. In case of ties, we select the FV that has the best average R-precision, using the values of Figure 3 as reference. This purity selection method tries to measure the "coherence" of the retrieved objects in the first positions of the ranking. Our hypothesis is that a good FV will rank objects from the same model class at the first positions of the ranking. On the other hand, if a FV ranks objects from different model classes in the first positions, then one can assume that the answer is not coherent and hence the FV is not suitable for this query.

Figure 6 shows a comparison between the purity selection technique and the best FVs, using the set of classified objects as the set $Q$ for the purity computation. We tested values for $k$ from 3 up to 10 , noticing only small variations in the results (in the figure, we show the results using $k=7$ ). With the purity selection method, we obtained an improvement of $21 \%$ in R-precision compared to the best 
average single $\mathrm{FV}$, which is a significant gain in retrieval effectiveness.

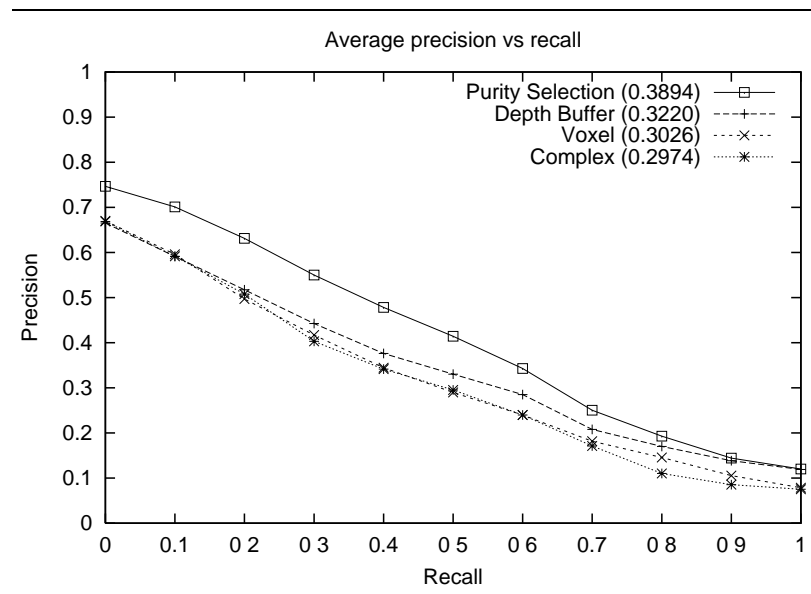

Figure 6. Comparison between the purity selection method and the best feature vectors.

To discard that the selection of the model classes could have some influence on the obtained results, we qualitatively validated the obtained results running a cross-validation test [6]. We divided a subset of the classified objects into two equally sized groups: A query set and a test set. The query set was used as in the described experimental framework. For computing the purity values we only used the test set, which was considered to be "out" of the database. That is, the test set was not considered for computing the effectiveness of the search system. Partitioning of the classified set was randomly performed, and we average over 100 random partitions. The results of the cross-validation test confirm the significant improvement of our purity selection method.

\section{Combinations of feature vectors}

The retrieval performance analysis in Section 3.2 suggests that there exist a number of FVs that achieve good average retrieval performance on the majority of query classes, but that there is no clear winner among them. Instead, the individual FVs have different strengths and weaknesses, and they represent complementary information regarding the similarity among 3D objects.

Because FVs capture different aspects and characteristics of the models, we propose to use combinations of $F V s$ for further improving the retrieval effectiveness of the similarity search, thus avoiding the disadvantages of using a single feature, which captures only a single characteristic of an object.
So, how can different FVs be combined in a search system? A simple concatenation of all available FVs is not advisable due to effectiveness and efficiency reasons [9]: Effectiveness would degrade with the inclusion of FVs irrelevant to the queries, and efficiency would also degrade because of the large dimensionality of the resulting FV, a problem known as the curse of dimensionality. Therefore, it is an interesting problem to find whether there are combinations of FVs that are better suited for performing similarity search on certain object classes, or even if there are combinations that dominate others for all types of queries.

We propose two methods for combining FVs: An unweighted combination method, and a weighted combination method based on the purity concept.

\subsection{Unweighted combinations of feature vectors}

We ran retrieval experiments on all possible combinations of all FVs, using their best dimensionality given by Figure 3. This gives a total of $\sum_{k=2}^{6}\left(\begin{array}{l}6 \\ k\end{array}\right)=57$ different combinations of FVs. To construct the combinations, we use the sum of the unweighted normalized distances.

Definition 2 The unweighted normalized combined distance $d^{c}$ is defined as:

$$
d^{c}(q, o)=\sum_{i=1}^{N} b_{c_{i}} \frac{d_{i}(q, o)}{\operatorname{dmax}_{i}(q)}
$$

where $N$ is the total number of $F V s, b_{c_{i}}$ is a binary variable that indicates whether $F V f_{i}$ is included in combination $c, d_{i}(q, o)$ is the distance of a query object $q$ from another object o under $f_{i}$, and $\operatorname{dmax}_{i}(q)$ is the maximum distance of object $q$ to any other object in the database as measured by $f_{i}$.

As in the single FVs experiments, the combined distance $d^{c}$ gives the ranking of objects w.r.t. a query $q$. The unweighted combination approach treats all FVs of the combination as equally important in determining the ranking.

Table 2 shows the average effectiveness of the best combinations of FVs in terms of R-precision and combination cardinality. The results confirm our assumption that there exist FV combinations that significantly improve the retrieval performance over the best single FV (depth buffer) in the average case. The maximum R-precision value reached on average over all query classes by a combination amounts to $42.89 \%$, which is equal to an improvement of more than $33 \%$ compared to the performance of the depth buffer. This best combination is composed of all six FVs. The largest improvement occurs when changing from the single to the 2-combination case (voxel and complex). The improvement increases further with combination cardinality, but the increment becomes smaller as we add more FVs to the combination. For the last one, the improvement in effectiveness 


\begin{tabular}{|c|c|c|}
\hline Comb. \# & R-precision & Feature vectors \\
\hline 1 & 0.3220 & Depth Buffer \\
\hline 2 & 0.3803 & Voxel, Complex \\
\hline 3 & 0.4108 & $\begin{array}{l}\text { Depth Buffer, Voxel, } \\
\text { Complex }\end{array}$ \\
\hline 4 & 0.4200 & $\begin{array}{l}\text { Depth Buffer, Voxel, } \\
\text { Complex, Silhouette }\end{array}$ \\
\hline 5 & 0.4287 & $\begin{array}{l}\text { Depth Buffer, Voxel, } \\
\text { Complex, Silhouette, } \\
\text { Harmonics 3D }\end{array}$ \\
\hline & & \\
\hline
\end{tabular}

Table 2. Average R-precision for the best unweighted combinations of feature vectors.

is negligible. Figure 7 shows the precision vs. recall curves for the best unweighted combinations.

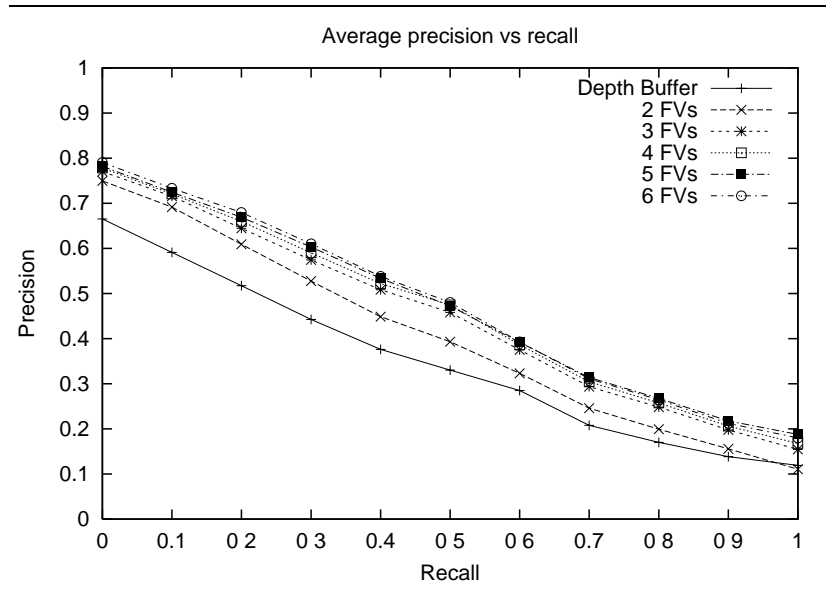

Figure 7. Average precision vs. recall for the best feature vector and the best unweighted combinations for an increasing numbers of feature vectors.

Note that we also performed a much larger series of experiments considering combinations of up to nine FVs. In these experiments we found that the retrieval effectiveness even starts to decrease when adding more FVs after a certain saturation point has been reached.

\subsection{Weighted combination of feature vectors}

A further improvement over the unweighted combination of FVs can be achieved by assigning weights to each $\mathrm{FV}$ in the combined distance, because it is expected that not all FVs are equally relevant to all queries, and using a non- suitable FV can even lower the effectiveness of the search. We tested all possible weightings for the combination of the six FVs using three different weight values $(0,1,2)$, resulting in $3^{6}-1=728$ different combinations. We call this approach fix-weighted combination, because each combination uses the same set of weight values $w=\left\{w_{1}, \ldots, w_{6}\right\}$ for all queries. The weights are assigned to each FV in the order given by Figure 3 (e.g., $w_{1}$ corresponds to depth buffer, $w_{2}$ corresponds to voxel, and so on).

Definition 3 The fix-weighted combined distance is defined as:

$$
d_{\text {fix-weighted }}(q, o)=\sum_{i=1}^{N} w_{i} \frac{d_{i}(q, o)}{\operatorname{dmax}_{i}(q)}
$$

The experimental results show that the set of weights $w^{*}=\{2,1,2,0,1,1\}$ provides the best performance. The precision vs. recall plot is shown in Figure 8. While this weight vector provides excellent retrieval performance, it is expected to be highly related to our database. Thus, it will probably not be useful for another 3D objects database, because the optimal average weighting may be different. Moreover, in a dynamic database, it is not possible to determine the best weighting factors by experimentally analyzing all combinations of weighting factors for all possible queries. All these negative attributes make this approach unpractical for real-world applications.

To overcome these problems, we propose another combination technique. The purity-weighted combination uses a dynamically determined weighting scheme based on the purity concept. The new combined distance is defined as follows.

Definition 4 The purity-weighted combined distance is defined as:

$$
d_{p \text {-weighted }}(q, o)=\sum_{i=1}^{N}\left(\operatorname{purity}\left(f_{i}, q, k\right)-1\right) \frac{d_{i}(q, o)}{\operatorname{dmax}_{i}(q)}
$$

Figure 8 shows the average precision versus recall figures for both weighted combinations of FVs methods and each single FV. For the purity value computation, we show the results using $k=4$ (results using values between 3 and 10 are all similar). For the fix-weighted combination, we show the result of using $w^{*}$ as the fixed weighting scheme. The improvements obtained with the weighted combinations (38\% improvement in R-precision compared with the best single FV) is far superior to the improvements obtained when using a different single FV. Both weighting combination methods have almost the same effectiveness on average, but notice that in the case of the fix-weighted combination we had to perform a brute force search to find the best weighting values (which, probably, are not optimal w.r.t. a different database). In contrast, the purity-weighting 
method automatically determines the weights for each FV depending on the query object.

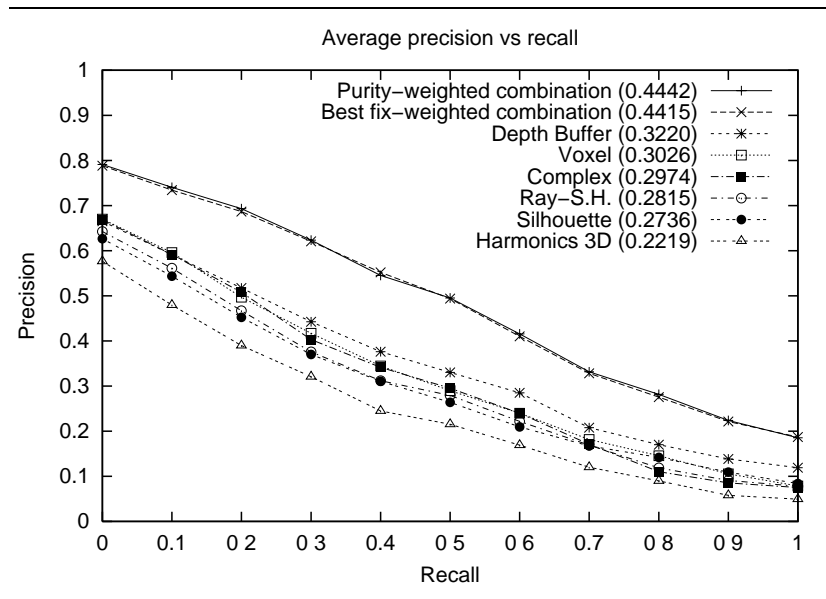

Figure 8. Average precision vs. recall figures for weighted combinations and its components.

Figure 9 shows the average precision versus recall figures for the Formula 1 cars model class, where significant improvements in retrieval effectiveness occur with the purity-weighted combination of FVs over the best single FV (39\% in terms of R-precision). The method sustains a nearperfect precision level for almost all recall levels, with just a small degradation for the very high recall levels.

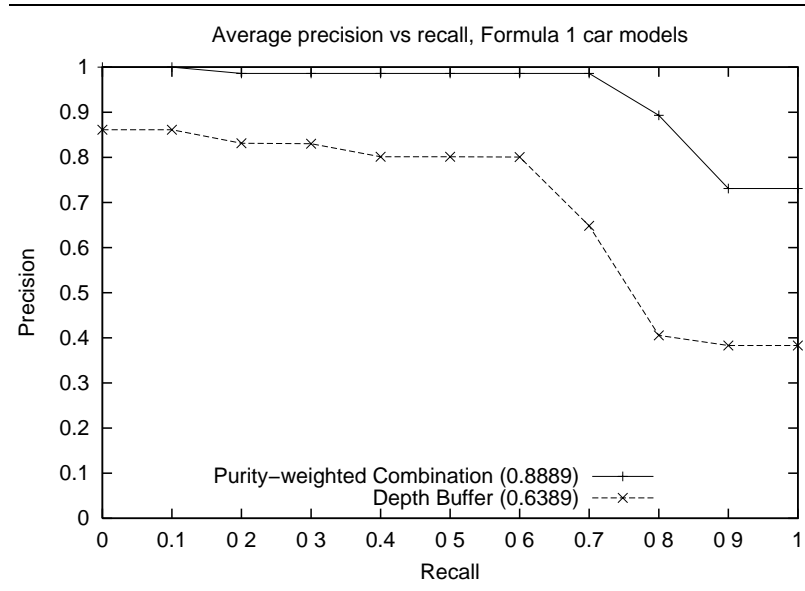

Figure 9. Average precision vs. recall figures, purity-weighted combination of feature vectors, F-1 cars model class.

\begin{tabular}{|l|c|c|}
\hline Method & R-precision & Improvement \\
\hline Best single feature & 0.3220 & $0 \%$ \\
vector & 0.3894 & $21 \%$ \\
Purity selection & 0.4289 & $33 \%$ \\
Best unweighted & & \\
combination & 0.4415 & $37 \%$ \\
Best fix-weighted & & \\
combination & 0.4442 & $38 \%$ \\
Purity-weighted & & \\
combination & \\
\hline
\end{tabular}

Table 3. Improvements in effectiveness obtained with the proposed techniques.

\section{Conclusions and future work}

In this paper, we described the challenges involved in the implementation of a content-based 3D similarity search system. Our first contribution is the proposal of a new selection method based on our purity concept, which determines the FV to be used for retrieval. The results show a significant effectiveness improvement over the best single FV. Secondly, we established that combinations of FVs may be highly beneficial for improving retrieval effectiveness in a 3D search system. We proposed to use a dynamically weighted combination of FVs based on the purity measure, thus avoiding the disadvantages of using just a single FV for the search. The experimental results show that the weighted combination of FVs further improves the retrieval effectiveness of the search system, and this improvement is far superior to the improvement obtained when switching from one type of FV to another. Table 3 summarizes the improvements obtained with the proposed techniques. Note that an improvement of $38 \%$ in effectiveness is very significant compared to the improvements of recently proposed FVs, which in most cases is in the order of $5 \%$ over previous methods.

It is worth noting that the proposed techniques are general and not restricted to $3 \mathrm{D}$ objects, and that they can be used with any multimedia data type (images, audio, etc.). Future work involves further researching query dependent feature selection and combination methods for other types of multimedia data. The final goal is to define a query processor that does not need a classified set of objects but is still capable of determining a good combination of feature vectors given a query object. It is also an open issue, how the efficiency of the search system can be improved. The need for appropriate indexing techniques, considering the very high dimensionality of the combined feature vectors (hundreds of dimensions) is obvious. 


\section{Acknowledgments}

This work was partially funded by the Deutsche Forschungsgemeinschaft (DFG), Projects No. KE 740/6-1 and No. SA 449/10-1, within the strategic research initiative "Distributed Processing and Delivery of Digital Documents" (V3D2), SPP 1041. It was also partially funded by the Information Society Technologies program of the European Commission, Future and Emerging Technologies under the IST-2001-33058 PANDA project (2001-2004). The first author is on leave from the Department of Computer Science, University of Chile.

\section{References}

[1] M. Ankerst, G. Kastenmüller, H.-P. Kriegel, and T. Seidl. 3D shape histograms for similarity search and classification in spatial databases. In 6th Intl. Symp. on Advances in Spatial Databases, LNCS 1651, pages 207-226. Springer, 1999.

[2] M. Ankerst, H.-P. Kriegel, and T. Seidl. A multistep approach for shape similarity search in image databases. IEEE Trans. on Knowledge and Data Engineering, 10(6):9961004, 1998.

[3] R. Baeza-Yates and B. Ribeiro-Neto. Modern Information Retrieval. Addison-Wesley, 1999.

[4] D.-Y. Chen, X.-P. Tian, Y.-T. Shen, and M. Ouhyoung. On visual similarity based 3D model retrieval. In Proc. Eurographics, 2003.

[5] T. Funkhouser, P. Min, M. Kazhdan, J. Chen, A. Halderman, D. Dobkin, and D. Jacobs. A search engine for 3D models. ACM Trans. on Graphics, 22(1):83-105, 2003.

[6] J. Han and M. Kamber. Data mining: concepts and techniques. Morgan Kauffman, 2001.

[7] M. Heczko, D. Keim, D. Saupe, and D. Vranic. Methods for similarity search on 3D databases. Datenbank-Spektrum, 2(2):54-63, 2002. In German.

[8] M. Hilaga, Y. Shinagawa, T. Kohmura, and T. Kunii. Topology matching for fully automatic similarity estimation of 3D shapes. In Proc. ACM Intl. Conf. on Computer Graphics and Interactive Techniques, pages 203-212. ACM Press, 2001.

[9] A. Hinneburg, C. Aggarwal, and D. Keim. What is the nearest neighbor in high dimensional spaces? In Proc. 26th Intl. Conf. on Very Large Databases, pages 506-515. Morgan Kaufmann, 2000.

[10] B. Horn. Extended Gaussian image. Proceedings of the IEEE, 72(12):1671-1686, 1984.

[11] T. Kato, M. Suzuki, and N. Otsu. A similarity retrieval of 3D polygonal models using rotation invariant shape descriptors. In Proc. IEEE Intl. Conf. on Systems, Man, and Cybernetics, pages 2946-2952, 2000.

[12] M. Kazhdan, T. Funkhouser, and S. Rusinkiewicz. Rotation invariant spherical harmonic representation of 3D shape descriptors. In Proc. Eurographics/ACM SIGGRAPH Symp. on Geometry Processing, pages 156-164. Eurographics Association, 2003
[13] H.-P. Kriegel, S. Brecheisen, P. Kröger, M. Pfeifle, and M. Schubert. Using sets of feature vectors for similarity search on voxelized CAD objects. In Proc. ACM Intl. Conf. on Management of Data (SIGMOD'03), pages 587598. ACM Press, 2003.

[14] G. Leifman, S. Katz, A. Tal, and R. Meir. Signatures of 3D models for retrieval. In Proc. 4th Israel-Korea Bi-National Conf. on Geometric Modeling and Computer Graphics, pages 159-163, 2003.

[15] R. Ohbuchi, T. Otagiri, M. Ibato, and T. Takei. Shapesimilarity search of three-dimensional models using parameterized statistics. In Proc. 10th Pacific Conf. on Computer Graphics and Applications, pages 265-274, 2002.

[16] R. Osada, T. Funkhouser, B. Chazelle, and D. Dobkin. Shape distributions. ACM Trans. on Graphics, 21(4):807-832, 2002.

[17] E. Paquet, M. Murching, T. Naveen, A. Tabatabai, and M. Rioux. Description of shape information for 2-D and 3-D objects. Signal Processing: Image Communication, 16:103$122,2000$.

[18] H. Sundar, D. Silver, N. Gagvani, and S. Dickinson. Skeleton based shape matching and retrieval. In Proc. Intl. Conf. on Shape Modeling and Applications, pages 130-142, 2003.

[19] J. Tangelder and R. Veltkamp. Polyhedral model retrieval using weighted point sets. Intl. Journal of Image and Graphics, 3(1):209-229, 2003.

[20] G. Taubin and D. Cooper. Recognition and positioning of rigid objects using algebraic moment invariants. In Proc. SPIE Conf. on Geometric Methods in Computer Vision, 1991.

[21] D. Vranic. An improvement of rotation invariant 3d shape descriptor based on functions on concentric spheres. In Proceedings of the IEEE Intl. Conf. on Image Processing (ICIP 2003), volume 3, 2003.

[22] D. Vranić and D. Saupe. 3D model retrieval with spherical harmonics and moments. In Proc. DAGM-Symp., LNCS 2191, pages 392-397. Springer, 2001.

[23] D. Vranić and D. Saupe. 3D shape descriptor based on 3D fourier transform. In Proc. EURASIP Conf. on Digital Signal Processing for Multimedia Communications and Services (ECMCS'01), pages 271-274. Comenius University, 2001.

[24] D. Vranic and D. Saupe. A feature vector approach for retrieval of 3D objects in the context of MPEG-7. In Proc. Intl. Conf. on Augmented, Virtual Environments and ThreeDimensional Imaging (ICAV3D’01), pages 37-40, 2001.

[25] D. Vranić and D. Saupe. Description of 3D-shape using a complex function on the sphere. In Proc. IEEE Intl. Conf. on Multimedia and Expo, pages 177-180, 2002.

[26] D. Vranić, D. Saupe, and J. Richter. Tools for 3D-object retrieval: Karhunen-Loeve transform and spherical harmonics. In Proc. IEEE 4th Workshop on Multimedia Signal Processing, pages 293-298, 2001.

[27] T. Zaharia and F. Prêteux. Three-dimensional shape-based retrieval within the MPEG-7 framework. In Proc. SPIE Conf. on Nonlinear Image Processing and Pattern Analysis XII, volume 4304, pages 133-145, 2001. 